\title{
Summary, Conclusions and Recommendations
}

\author{
Rosemary F. G. Wyse \\ Department of Physics \& Astronomy, Johns Hopkins University, \\ Baltimore, MD 21218, USA \\ email: wyse@pha.jhu.edu
}

\section{Legacies of Bengt Strömgren}

Studying the disk of the Milky Way in its cosmological context, as reflected in the title of this conference, was pioneered by Bengt Strömgren, in whose honour we are gathered here. A significant legacy of his is the understanding that advances are achieved by making connections among fields that are tenuously related on initial inspection. When the tools to achieve this were not available, Bengt Strömgren developed the necessary techniques, such as Strömgren photometry to quantify the local stellar population.

This points to another legacy of Bengt Strömgren: the appreciation that many approaches, tools and techniques provide complementary information, and one should use them all. Examples, all of which formed lively discussions here, include

- The direct study of systems at high redshift, where one is limited to interpretation of the integrated light, compared to more detailed study in local systems of individual old stars, which formed long ago.

- Numerical simulations compared to analytic theory

- Indeed, theoretical predictions confronted with observations

- Properties of stellar populations compared with those of the gaseous components

- The different observational evidence provided by spectroscopy, photometry and astrometry

\section{Connections}

The presentations and discussions at this meeting identified several areas where connections are apparent, albeit that we are not yet clear of the nature of the connection, or the physics behind the connection. As in any lively interchange of ideas, as many questions were raised as were answered.

\subsection{High redshift $\Leftrightarrow$ low redshift}

What are the descendants of the turbulent '(thick?) disk' galaxies at redshifts $\sim 2$ ? These have very high inferred gas densities and star formation rates; could this be more suggestive of bulge formation that of thick disk formation?

Where are the first stars (Population III), and their immediate descendants, stars that are enriched by Population III and should show distinctive elemental abundance patterns if predictions of a top-heavy stellar initial mass function are correct?

What were/are the first galaxies? Have they all been subsumed into larger systems? The limitation to integrated light in high redshift systems leads to inevitable degeneracies such as between the inferred star formation rate and stellar IMf, and between age and metallicity. The more detailed information available for resolved stellar populations locally allows one to break these degeneracies. 


\subsection{Local star formation $\Leftrightarrow$ global star formation efficiency}

How effective is feedback from supernovae? Type Ia and Type II? Is it always negative? Do supernovae actually act coherently to drive winds or do they simply lower the star formation efficiency within bound gas clouds? What sets the constant of proportionality in the scaling relation between gas content and star formation rate (the 'Schmidt-Kennicutt' law)? Why does this relation remain valid over a very large range of physical conditions and scales? The molecular gas fraction is clearly important in determining star formation rates and efficiencies, given that stars are observed forming in molecular clouds. What sets this fraction?

Supernovae - both core collapse, Type II, and thermonuclear, Type Ia - clearly act to disperse metals, which aid cooling and later star formation. But how this mixing occurs within a galaxy's interstellar medium, and what fraction of these metals are mixed into the intergalactic medium, remain much debated.

\subsection{Bulge formation $\Leftrightarrow$ (thick) disk formation}

At least in the Milky Way Galaxy, these two stellar components have similar stellar age distributions, being dominantly old, typically 10-12 Gyr. The more limited data for external galaxies also suggest old ages, certainly significantly older than the mean age of stars in thin disks.

Both components also have relatively high metallicity, which, combined with the old mean age and narrow age range, implies (relatively) high star formation rates and enrichment efficiency. This points to high gas densities and a deep potential well and could be suggestive of the turbulent stellar systems at redshift $\sim 2$ (look-back time of 10.3 Gyr in the 'concordance' cosmology) referred to above. It does not suggest significant mass build-up in the form of stars accreted from former satellite galaxies. Mergers can however drive gas to the central regions, to fuel a dissipative starburst, and perhaps a merger both created the thick disk and triggered bulge star formation.

The (present) thin disk would be largely established later, plausibly in the form of gas accretion/infall. This provides a connection to another 'connection' below, gas $\Leftrightarrow$ stars, as several speakers pointed out the uncertainties in gas reservoir and accretion rates.

In all interpretations of stellar populations, age determinations are critical (and one of the goals of Strömgren photometry).

\subsection{First stars $\Leftrightarrow$ stars now}

Arguments based on cooling inefficiency and slow rates of molecular gas formation at zero metallicity lead to the expectation that the first stars should have a mass function that is biased to massive stars, perhaps even to the exclusion of any stars below the most massive stars seen today. However, the same arguments lead to the expectation that the stellar IMF is invariant above some low threshold metallicity. Observational evidence for an invariant IMF comes from star counts within the Milky Way and its companion galaxies: these are consistent with the same low-mass IMF in the central bulge (high metallicity, high SFR, high density) as in globular clusters (low metallicity) as in the Ursa Minor dwarf spheroidal galaxy (low metallicity, low SFR, low density), as in the local disk now (high metallicity, moderate SFR, moderate density).

The past high-mass IMF is constrained by the elemental abundance patterns in the long-lived, low-mass stars that those high-mass stars enriched: yields of $\alpha$-elements from Type II supernovae tend to increase with the (main sequence) mass of the progenitor, while the iron yield varies little with progenitor mass. These trends result in higher predicted values of $[\alpha / \mathrm{Fe}]$ in stars enriched by massive stars with an IMF biased to the highest mass, massive stars. Observations of elemental abundances in halo stars down to 
below one-thousandth of the solar iron abundance have revealed very little scatter in the value of the 'Type II' plateau: this low scatter implies that independent of metallicity, the same massive-star IMF was sampled - and that there was good mixing, and that the entire IMF was sampled, both of these points themselves posing puzzles.

\subsection{Substructure $\Leftrightarrow$ galactic scale structure}

Substructure, whether in the form of CDM pre-galactic systems, dynamical instabilities or even black holes, can make its presence known in several ways. Disk-heating associated with the merging of satellite galaxies is the focus of most discussion, but gravitational interactions with a various classes of substructure could e.g. disrupt wide binaries, disturb thin tidal arms from disrupting globular clusters and satellites, cause microlensing events etc. Keeping substructure dark does not hide it, unless it is kept far away from any stellar component.

Interactions of stars with giant molecular clouds and spiral arms have long been implicated in underlying the age-velocity dispersion relation for thin disk stars (the thick disk is too hot for this mechanism to be responsible for its vertical structure), albeit imperfectly understood. It has recently become clear that stellar disks are rather dynamic, and that resonances within the disk can interact with disk stars to create 'moving groups', consisting of stars with a common motion but often a rather broad age range and metallicity distribution. These need to be distinguishable and distinguished from debris from any putative satellite system accreted into the thin disk plane. Connecting large scales, resonances can induce radial migration of stars within the thin disk, over essentially a disk scale-length; this effect needs to be quantified.

Disk instability can also form a central bar, which in turn can buckle to form a bulge; this phenomenon has been know for years, but the duty cycle, prevelance, signatures and indeed criteria are still not well understood. How bars interact with a background dark halo is also a topic of much research, in particular whether or not the mass profile of the dark halo could be significantly modified. Interactions with substructure, such as satellites, bars, and spiral arms can cause transport of angular momentum on larger scales, and again the lively discussions point to an active research area.

Stellar streams in the (outer) stellar halo of the Milky Way and other galaxies are testament to interactions between substructure and the host system. Dynamical times are longer in the lower density outer parts, allowing streams in coordinate space to persist and be identified. Indeed, leading and trailing debris from more than one periGalactic passages of the Sagittarius dwarf spheroidal can be traced, and this one system accounts for a significant fraction of the detected substructure in the outer halo. A similar situation may occur in M31, where one satellite can in principle provide all the observed structure in the halo.

\subsection{Dark matter $\Leftrightarrow$ light matter}

The Tully-Fisher relation is a fundamental observational relationship that all theories of galaxy formation need to reproduce from first principals. The fewer 'baryonic physics' knobs that are tuned, the better. However, there is some observational evidence for different star-formation modes (e.g. starburst vs quiescent; stochastic $v s$ triggered) in different mass/redshift haloes, and this needs to be understood.

The motions of light matter (stars and gas) within dominant dark matter haloes can be used to constrain the nature of dark matter by, e.g., identification of the signatures of merging. In this context, large surveys of Galactic stars are very important (as recognized by Bengt Strömgren!). 


\subsection{Gas $\Leftrightarrow$ stars}

The 'Schmidt-Kennicutt Law' of star formation clearly lacks understanding. While one can straightforwardly argue that any scaling of star formation rate should involve the amount of fuel (gas) divided by a timescale (dynamical time, going like one over the square root of gas volume density for self-gravitating gas), it is neither observationally nor theoretically clear whether this criterion should invoke the molecular gas, or the atomic gas, or the total gas, and whether it should be volume density or surface density, or one power or two (collisionally induced?) and on what scale it is appropriate to average.

Fuel for star formation, i.e. gas, apparently needs to be added to galaxies as they evolve, and there was much discussion of accretion and gas cycling among different physical phases. The evidence for accretion depends on analysis of high velocity clouds in HI maps of our own and external galaxies, and is complicated by the possibility of largescale 'fountain' effects and distance uncertainties. It remains unclear what forms the reservoir - is it the 'missing baryons' postulated to be in a diffuse, warm 'cosmic web'? The accretion rate as a function of time and place is poorly constrained, as are the angular momentum content and metallicity of any accreted gas. Whether or not there are always associated dark matter lumps, or one has smooth dissipational settling of baryonic material is unclear.

\subsection{Chemical evolution $\Leftrightarrow$ dynamical evolution}

On large scales, the existence of the well-established mass-metallicity relation for galaxies of a range of morphological types argues strongly that chemical evolution and dynamics are linked - the most straightforward explanation is that the bulk of stars formed in galaxies with a potential well depth very similar to the one in which the stars are today.

Within galaxies, one cannot ignore the effects of dynamical instabilities and resonances in the thin disk when calculating e.g. chemical abundance gradients, and stellar agevelocity dispersion relations. There could be significant radial re-arrangement and mixing of stars, in addition to gas flows.

It is also clear that one cannot model chemical evolution of the galaxy assuming an extension of the Simple Model that maintains homogeneity and a one-to-one correspondence between time and metallicity, even for the solar neighbourhood. Stochastic star formation, possibly reviving metal-enhanced star formation ideas, should be incorporated. However, one needs to maintain the remarkable lack of scatter in elemental abundance patterns in each of the different stellar components, at all metallicities.

Cosmological boundary conditions should also be applied, rather than invoking gas inflow/outflow ad hoc, with little or no consideration to angular momentum conservation or cooling times. Progress is being made, and this is a very active field, as envisaged by Bengt Strömgren.

\section{Conclusions \& Recommendations}

Bringing together people from across the larger astrophysical community with the same 'big picture' science goals, as achieved with this meeting, is very fruitful, and certainly fosters the discussions and connections among researchers that is needed to make progress. I recommend that we should repeat this gathering!

\section{Acknowledgements}

I would like to thank Birgitta Nordström and Johannes Andersen for their extraordinary commitment and enthusiasm that played a pivotal role in the success of this meeting. All the members of the SOC and LOC are also due a vote of thanks. 